\title{
A multiwavelength study of the star forming region IRAS 18544+0112
}

\author{
M. E. Ortega ${ }^{1}$, S. Paron $^{1}$, S. Cichowolski ${ }^{1}$, M. Rubio ${ }^{2}$, G. Castelletti ${ }^{1}$, and G. Dubner ${ }^{1}$ \\ 1 Instituto de Astronomía y Física del Espacio (IAFE), CC 67, Suc. 28, 1428 Buenos Aires, Argentina \\ e-mail: mortega@iafe.uba.ar \\ 2 Departamento de Astronomía, Universidad de Chile, Casilla 36-D, Santiago, Chile
}

Received 18 June 2009 / Accepted 6 November 2009

\section{ABSTRACT}

\begin{abstract}
Aims. This work aims at investigating the molecular and infrared components in the massive young stellar object (MYSO) candidate IRAS $18544+0112$. The purpose is to determine the nature and the origin of this infrared source.

Methods. To analyze the molecular gas towards IRAS 18544+0112, we have carried out observations in a $90^{\prime \prime} \times 90^{\prime \prime}$ region around $l=$ $34.69, b=-0.65$, using the Atacama Submillimeter Telescope Experiment (ASTE) in the ${ }^{12} \mathrm{CO} J=3-2,{ }^{13} \mathrm{CO} J=3-2, \mathrm{HCO}^{+} J=4-3$ and CS $J=7-6$ lines with an angular resolution of $22^{\prime \prime}$. The infrared emission in the area has been analyzed using 2 MASS and Spitzer public data.

Results. From the molecular analysis, we find self-absorbed ${ }^{12} \mathrm{CO} J=3-2$ profiles, which are typical in star forming regions, but we do not find any evidence of outflow activity. Moreover, we do not detect either $\mathrm{HCO}^{+} J=4-3$ or CS $J=7-6$ in the region, which are species normally enhanced in molecular outflows and high density envelopes. The ${ }^{12} \mathrm{CO} J=3-2$ emission profile suggests the presence of expanding gas in the region. The Spitzer images reveal that the infrared source has a conspicuous extended emission bright at $8 \mu \mathrm{m}$ with an evident shell-like morphology of $\sim 1.5$ in size $(\sim 1.4 \mathrm{pc}$ at the proposed distance of $3 \mathrm{kpc})$ that encircles the $24 \mu \mathrm{m}$ emission. The non-detection of ionized gas related to IRAS 18544+0112 together with the fact that it is still embedded in a molecular clump suggest that IRAS $18544+0112$ has not reached the UCHII region stage yet. Based on near infrared photometry we search for YSO candidates in the region and propose that 2MASS $18565878+0116233$ is the infrared point source associated with IRAS 18544+0112. Finally, we suggest that the expansion of a larger nearby HII region, G034.8-0.7, might be related to the formation of IRAS $18544+0112$.
\end{abstract}

Key words. ISM: molecules - HII regions - stars: formation

\section{Introduction}

Star formation processes start when a pressure-bounded, selfgravitating molecular clump becomes gravitationally unstable. As summarized by Whitworth et al. (1994a,b), the action of an expanding nebula can produce gravitationally unstable shocked layers of interstellar gas. For example, the expansion of an HII region can sweep up the surrounding molecular gas into a dense shell, which then fragments and forms new massive stars ("collect and collapse" model; see Elmegreen \& Lada 1977). Several recent works support this model (e.g. Deharveng et al. 2005; Comerón et al. 2005; Zavagno et al. 2006; Pomarès et al. 2009). On the other hand, shockwaves from expanding windand/or supernova-driven superbubbles can also trigger cloud collapse and star formation, but at larger scales. Numerical studies (Vanhala \& Cameron 1998; Melioli et al. 2006) demonstrated that the effect caused by a passing shockwave mainly depends on the shock-type: close to the supernova remnant (SNR) the shockwave disrupts the ambient molecular clouds and thus terminates the star formation process; however, a little further away from the SNR the shock velocity decreases, and cloud collapse is possible if the right circumstances were given.

This work is part of a systematic study towards IR sources embedded in molecular condensations with evidence of being affected by SNRs shock fronts or expanding HII regions. In a previous work, Paron et al. (2009) studied the infrared (IR) source IRAS $18542+0114$ located near the border of the SNR
W44. They discovered that this source is probably a massive young stellar object (MYSO) located at the border of the HII region G034.8-0.7 which is evolving within a molecular cloud. In this work, we present a study of the neighboring source IRAS 18544+0112, another IR source embedded in the same molecular cloud. It is important to remark that the molecular cloud, the HII region G034.8-0.7 and the SNR W44 are not only located in the same region in the plane of the sky but also at the same distance from the Sun, at about $3 \mathrm{kpc}$ (corresponding to the kinematic velocity of $v_{\mathrm{LSR}} \sim 45 \mathrm{~km} \mathrm{~s}^{-1}$, Paron et al. 2009, and references therein).

The source investigated in this Paper, IRAS 18544+0112, was cataloged as a MYSO and a high mass protostellar object (HMPO) by Molinari et al. (1996) and Kumar \& Grave (2007), respectively. On the basis of the analysis of new molecular data and near- and mid-IR data, we present a multiwavelength study of the IR source aiming to discern its origin and evolutionary stage.

\section{IRAS $18544+0112$ and its environment}

Figure 1 shows a two-color IR image of the HII region G034.8-0.7. We show in green the Spitzer-IRAC $8 \mu \mathrm{m}$ emission and in red the Spitzer-MIPSGAL $24 \mu \mathrm{m}$ emission. Yellow corresponds to regions where both emissions overlap. The white contours represent the ${ }^{13} \mathrm{CO} J=1-0$ line emission distribution 


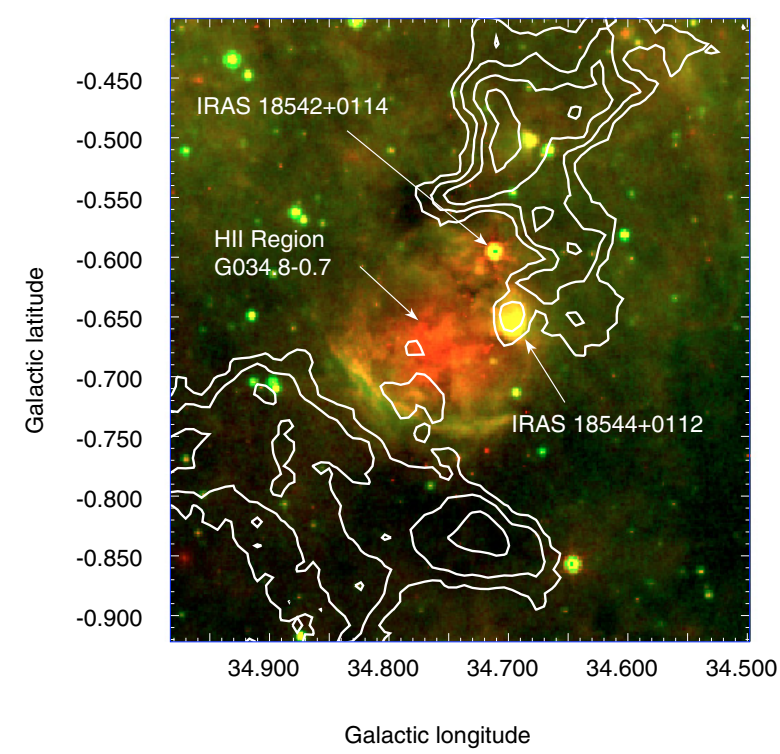

Fig. 1. Two-color image of the HII region G034.8-0.7 containing IRAS $18542+0114$ and IRAS $18544+0112$. Green is the Spitzer-IRAC $8 \mu \mathrm{m}$ emission and red is the Spitzer-MIPSGAL $24 \mu \mathrm{m}$ emission. White contours at $0.4,0.6,1$ and $1.2 \mathrm{~K}$ represent the ${ }^{13} \mathrm{CO} J=1-0$ line emission distribution integrated between the velocity range from 36 to $45 \mathrm{~km} \mathrm{~s}^{-1}$.

as extracted from the Galactic Ring Survey (GRS; Jackson et al. 2006) averaged over the velocity interval from 36 to $45 \mathrm{~km} \mathrm{~s}^{-1}$.

The $8 \mu \mathrm{m}$ emission, which arises mainly from the polycyclic aromatic hydrocarbons molecules (PAHs; Leger \& Puget 1984) and an underlying continuum attributed to very small grains, is observed enclosing the $24 \mu \mathrm{m}$ emission that is originated in the heated dust of the HII region G034.8-0.7. These molecules cannot survive inside HII regions and are located over the photodissociation regions (PDRs) that encircle the ionized gas (Cesarsky et al. 1996). The PDRs are the interphase zone between the ionized and molecular gas, and its presence evidences the interaction between them. A well defined $8 \mu \mathrm{m}$ arc-like structure is observed bordering the HII region G034.8-0.7 towards lower Galactic latitudes. It is important to note that the ionized region is partially bordered by two molecular clouds which are part of the giant molecular complex (GMC) G34.8-0.6. The morphology of the molecular clouds, as seen in the ${ }^{13} \mathrm{CO} J=1-0$ line, suggests that they are shaped by the action of the HII region.

On the other hand, IRAS 18544+0112, located at $(l, b)=\left(34^{\circ} 69,-0.65\right)$, appears as a bright yellow knot. From Fig. 1 it can be noticed that this IR source is located in a bulge shaped molecular gas condensation which is observed in the velocity interval from 36 to $45 \mathrm{~km} \mathrm{~s}^{-1}$.

Figure 2 shows a two-color image of IRAS 18544+0112, where green and red are the $8 \mu \mathrm{m}$ and $24 \mu \mathrm{m}$ emission, respectively. The observed $8 \mu \mathrm{m}$ emission distribution shows a shelllike structure of about 1.5 in size $(\sim 1.4 \mathrm{pc}$ at a distance of $3 \mathrm{kpc})$, bright filaments and diffuse emission. The emission at $24 \mu \mathrm{m}$ is observed mainly inside the shell-like structure.

Since most HMPOs present molecular outflows, we now analyze whether this phenomenon is taking place in the region.

The IRAC $4.5 \mu \mathrm{m}$ band contains lines that may be excited by high velocity shocks, such as those expected when protostellar outflows crash into the ambient ISM (Cyganowski et al. 2008) or

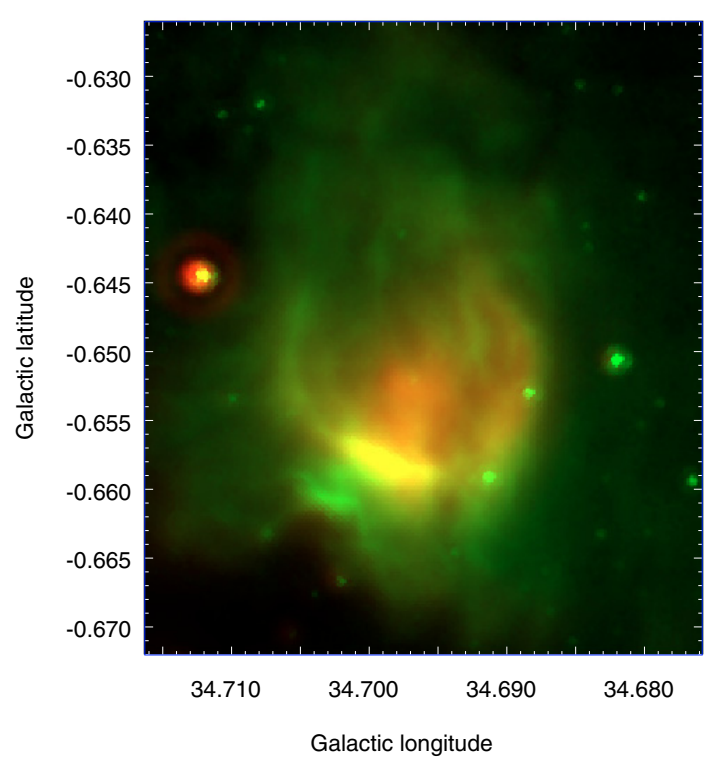

Fig. 2. The green is the Spitzer-IRAC $8 \mu \mathrm{m}$ emission and the red is the MIPSGAL emission at $24 \mu \mathrm{m}$ towards IRAS $18544+0112$.

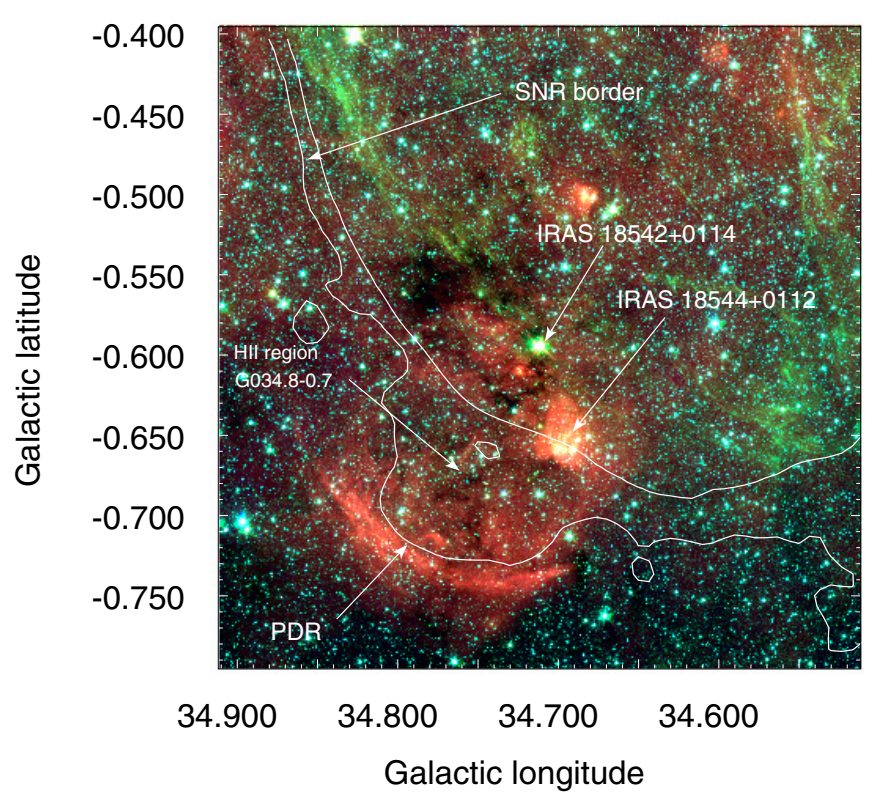

Fig. 3. Spitzer-IRAC three-color image $(3.5 \mu \mathrm{m}=$ blue, $4.5 \mu \mathrm{m}=$ green, and $8 \mu \mathrm{m}=$ red) of a region of $22^{\prime}$ in size. The contours correspond to the $1.4 \mathrm{GHz}$ radio continuum emission at 17 and $35 \mathrm{~K}$, which depict the border of the HII region G034.8-0.7 and the SE border of the SNR W44, respectively.

when a SNR interacts with a molecular cloud. We thus inspected the IRAC $4.5 \mu \mathrm{m}$ emission distribution in the area.

Figure 3 shows a Spitzer-IRAC three-color image of a square region of $22^{\prime}$ in size. The three IR bands presented are: $3.6 \mu \mathrm{m}$ (in blue), $4.5 \mu \mathrm{m}$ (in green) and $8 \mu \mathrm{m}$ (in red). The white contours represent the radio continuum emission at $1.4 \mathrm{GHz}$ as extracted from the VGPS (VLA Galactic Plane Survey; Stil et al. 2006), which depict the SE border of the SNR W44 and the border of the radio continuum emission associated with the HII region G034.8-0.7 encompassing the PDR. 
An inspection of Fig. 3 shows that the source IRAS $18542+0114$ studied by Paron et al. (2009), appears slightly extended in the $4.5 \mu \mathrm{m}$ emission (green). Such a characteristic is suggestive of a YSO origin for the emission, a condition that was confirmed by Paron et al. (2009) after discovering associated molecular outflows. Figure 3 also shows some extended diffuse filaments in this IRAC band, which are probably illuminating molecular gas shocked by the SNR. In the case of the source studied in this work, IRAS 18544+0112, it does not present significant emission in the $4.5 \mu \mathrm{m}$ band. Indeed, this source is clearly brighter at $8 \mu \mathrm{m}$ than at $4.5 \mu \mathrm{m}$, suggesting that there is no outflow activity in the region.

With the purpose to analyze the small scale distribution and dynamic of the molecular gas associated with IRAS $18544+0112$, we carried out observations of the ${ }^{12} \mathrm{CO}$, ${ }^{13} \mathrm{CO} J=3-2, \mathrm{HCO}^{+} J=4-3$, and CS $J=7-6$ lines towards a region of $90^{\prime \prime} \times 90^{\prime \prime}$ around this IR source using the Atacama Submillimeter Telescope Experiment (ASTE; Ezawa et al. 2004).

\section{New molecular observations}

The molecular observations were performed on June 25, 2008 with the $10 \mathrm{~m}$ ASTE Telescope. We used the CATS345 GHz band receiver, which is a two-single band SIS receiver remotely tunable in the LO frequency range of $324-372 \mathrm{GHz}$. We simultaneously observed ${ }^{12} \mathrm{CO} J=3-2$ at $345.796 \mathrm{GHz}$ and $\mathrm{HCO}^{+} J=4-3$ at $356.734 \mathrm{GHz}$, mapping a region of $90^{\prime \prime} \times 90^{\prime \prime}$ centered at the position of IRAS 18544+0112, $(l, b)=(34.69,-0.65)$. The mapping grid spacing was $10^{\prime \prime}$ and the integration time was $72 \mathrm{~s}$. per pointing. Additionally, we observed ${ }^{13} \mathrm{CO} J=3-2$ at $330.588 \mathrm{GHz}$ and $\mathrm{CS} J=7-6$ at $342.883 \mathrm{GHz}$ towards the center of the region. All the observations were performed in position-switching mode. The offposition was $(l, b)=\left(34.87,-0^{\circ} 14\right)$, which was checked to be free of emission.

We used the XF digital spectrometer with a bandwidth and spectral resolution set to $128 \mathrm{MHz}$ and $125 \mathrm{kHz}$, respectively. The velocity resolution was $0.11 \mathrm{~km} \mathrm{~s}^{-1}$ and the half-power beamwidth (HPBW) was $22^{\prime \prime}$ at $345 \mathrm{GHz}$. The system temperature varied from $T_{\text {sys }}=400$ to $700 \mathrm{~K}$. The typical rms noise (in units of $T_{\mathrm{mb}}$ ) ranged between 0.1 and $0.4 \mathrm{~K}$, and the main beam efficiency was $\eta_{\mathrm{mb}} \sim 0.65$.

The spectra were Hanning-smoothed to improve the signalto-noise ratio, and only linear or/and some third order polinomia were used for baseline fitting. The spectra were processed using the XSpec software package developed at the Onsala Space Observatory.

\section{Molecular analysis}

As it was shown in Fig. 1, IRAS 18544+0112 appears embedded in a molecular clump, which is part of the GMC G34.8-0.6.

Figure 4(left) shows the ${ }^{12} \mathrm{CO} J=3-2$ spectra obtained from a region of $90^{\prime \prime} \times 90^{\prime \prime}$ centered at the position of IRAS $18544+0112$ and Fig. 4 (right) presents a spectrum obtained towards the center of the region. Figure 5 displays an average spectrum of ${ }^{13} \mathrm{CO} J=3-2$ obtained towards the center of the clump at $(l, b)=\left(34.69,-0^{\circ} 65\right)$. All the ${ }^{12} \mathrm{CO}$ profiles in this region present a dip at $v \sim 44 \mathrm{~km} \mathrm{~s}^{-1}$. Such a narrow dip points to a self-absorption origin instead of two ${ }^{12} \mathrm{CO}$ emission components with very close kinematical velocities. We notice that the $v \sim 44 \mathrm{~km} \mathrm{~s}^{-1}$ dip is very close to the peak velocity of the

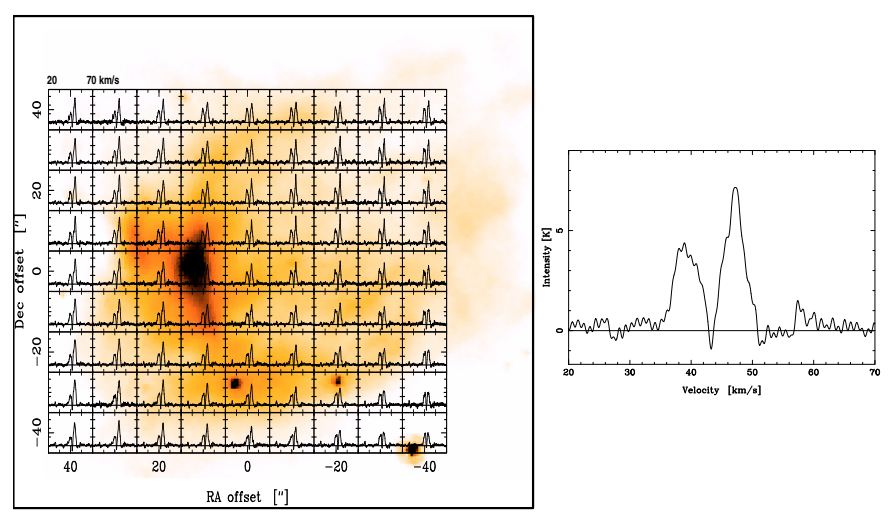

Fig. 4. Left: ${ }^{12} \mathrm{CO} J=3-2$ spectra obtained towards IRAS $18544+0112$. The velocity range of each spectrum is between 20 and $70 \mathrm{~km} \mathrm{~s}^{-1}$. The color scale corresponds to the emission at $8 \mu \mathrm{m}$ associated with IRAS 18544+0112, as extracted from Spitzer-IRAC. Right: ${ }^{12} \mathrm{CO} J=3-2$ spectrum towards the center of the region.

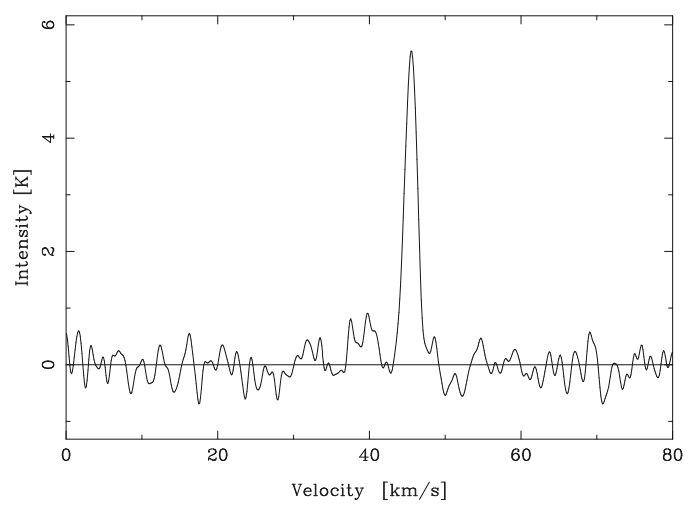

Fig. 5. ${ }^{13} \mathrm{CO} \quad J=3-2$ profile obtained towards the center of IRAS $18544+0112$.

${ }^{13} \mathrm{CO} J=3-2$ line, which is an optically thinner line. Such correspondence strongly suggests that the dip in the ${ }^{12} \mathrm{CO}$ profiles is in fact caused by self-absorption by less excited gas (see for example Zhou et al. 1993).

Since we are searching for indicators of active star formation in this IR source, the presence of this dip is interesting because it is known that the ${ }^{12} \mathrm{CO} J=3-2$ line has almost always been observed self-absorbed in star-forming regions (Johnstone et al. 2003).

Additionally, we note that the ${ }^{12} \mathrm{CO} J=3-2$ emission does not show any evidence of outflow activity in the region, which agrees with the faint $4.5 \mu \mathrm{m}$ emission mentioned in Sect. 2 . The non-detection of the $\mathrm{HCO}^{+} J=4-3$ and CS $J=7-6$, molecular species that are enhanced in the interphase layer between the outflows and the surrounding molecular core where a YSO is forming (Hogerheijde et al. 1998; Rawlings et al. 2004) points in the same direction.

On the other hand, Fig. 4 clearly shows that most of the ${ }^{12} \mathrm{CO}$ profiles in the region present a redshifted component brighter than the blueshifted one, suggesting that the molecular gas is expanding. This is because in an expanding cloud a line emission is composed by red and blueshifted photons. In the case of an optically thick line, such as the ${ }^{12} \mathrm{CO} J=3-2$, the redshifted photons will encounter fewer absorbing material (which is expanding outward) than would blueshifted photons and hence have greater probabilities of escape (e.g. Leung 1978; Zhou 1992; Lehtinen 1997). 
To estimate the physical parameters of the molecular clump where IRAS $18544+0112$ is embedded, we determine the line parameters of the ${ }^{13} \mathrm{CO} J=3-2$ line from a Gaussian fitting. We obtain: $T_{\mathrm{mb}} \sim(5.7 \pm 0.5) \mathrm{K}, V_{\mathrm{LSR}} \sim(45.4 \pm 0.4) \mathrm{km} \mathrm{s}^{-1}, \Delta v \sim(2.0 \pm$ $0.2) \mathrm{km} \mathrm{s}^{-1}$ and $I \sim(12 \pm 1) \mathrm{K} \mathrm{km} \mathrm{s}^{-1}$, where $T_{\mathrm{mb}}$ represents the peak brightness temperature, $V_{\mathrm{LSR}}$ the central velocity referring to the local standard of rest, $\Delta v$ the line width and $I$ the integrated line intensity. Errors are a formal $1 \sigma$ value for the model of the Gaussian line shape.

Using the ${ }^{13} \mathrm{CO} J=3-2$ and $J=1-0$ lines we calculated the ratio of the integrated line intensities $\left({ }^{13} R_{3-2 / 1-0}\right)$. The ${ }^{13} \mathrm{CO}$ $J=1-0$ line was extracted from the GRS and the $J=3-2$ line was convolved to the $J=1-0$ beam. We obtained ${ }^{13} R_{3-2 / 1-0} \sim 1$.

Assuming LTE conditions and a beam filling factor of 1 , which of course may not be completely true but allows us to make an initial guess, we use the ${ }^{12} \mathrm{CO} J=3-2$ emission towards the center of the analyzed region to estimate an excitation temperature, $T_{\mathrm{ex}}$. As noticed above, this emission appears self-absorbed, showing a dip between the blue and red emission components. Thus, we use an average between the $T_{\mathrm{mb}}$ of both components to obtain $T_{\text {ex }} \sim 15 \mathrm{~K}$. Using this factor and the parameters obtained for the ${ }^{13} \mathrm{CO} J=3-2$, we derive an optical depth for this line of $\tau^{13} \sim 0.2$ and a ${ }^{13} \mathrm{CO}$ column density of $N\left({ }^{13} \mathrm{CO}\right) \sim 2.2 \times 10^{15} \mathrm{~cm}^{-2}$. Adopting the ${ }^{12} \mathrm{CO}$ and ${ }^{13} \mathrm{CO}$ relationships of $N\left(\mathrm{H}_{2}\right) / N\left({ }^{12} \mathrm{CO}\right)=10^{5}$ and $N\left({ }^{12} \mathrm{CO}\right) / N\left({ }^{13} \mathrm{CO}\right)=89$ (Guan et al. 2008) and taking into account that ${ }^{13} R_{3-2 / 1-0}=$ 1 as estimated above, we obtain an $\mathrm{H}_{2}$ column density of $N\left(\mathrm{H}_{2}\right) \sim 2.0 \times 10^{21} \mathrm{~cm}^{-2}$. Finally, assuming a spherical geometry for the clump as seen in the ${ }^{13} \mathrm{CO} J=1-0$ line (Fig. 1-left) with a radius of $\sim 0.7 \mathrm{pc}$, we estimate a mass and a volume density of $\sim 1.7 \times 10^{2} M_{\odot}$, and $\sim 3.2 \times 10^{3} \mathrm{~cm}^{-3}$, respectively.

\section{Is IRAS 18544+0112 an ultracompact HII region?}

In view of the lack of outflow activity in the area, a possible explanation is that IRAS $18544+0112$ is an evolved high-massive protostellar object that has finished its accretion phase. Since the next step in the life of these objects is to develop an ultracompact HII (UCHII) region we looked for the presence of ionized gas. The fact that this source lies in the same region of the sky than the SNR W44 and the HII region G34.7-0.8, makes it difficult to discern whether there is radio emission related to IRAS 18544+0112. An inspection of the $1420 \mathrm{MHz}$ image obtained from the VGPS does shows the presence of emission at the position of IRAS $18544+0112$, but its extended morphology suggests that it is most probably related to G 34.7-0.8. To avoid the contamination of extended radio sources, we have inspected the $1420 \mathrm{MHz}$ radio continuum image obtained from the NRAO VLA Sky Survey (NVSS; Condon et al. 1998). No radio continuum emission is detected at the position of IRAS $18544+0112$. This agrees with the null detection reported by Hughes \& MacLeod (1994) in the direction of this source.

From the cataloged IRAS fluxes of IRAS 18544+0112 and assuming a distance of $3 \mathrm{kpc}$, we estimated the corresponding IR luminosity $L_{\mathrm{IR}}$ and dust temperature $T_{\mathrm{d}}$. The IR luminosity was estimated on the basis of the four-bands IRAS measurements $(12,25,60$ and $100 \mu \mathrm{m})$ following Chan \& Fich (1995) as $L_{\mathrm{IR}}\left(L_{\odot}\right)=1.58 S_{\mathrm{IR}}(\mathrm{Jy}) D^{2}(\mathrm{kpc})$, where $S_{\mathrm{IR}}$ is the integrated flux given by $S_{\mathrm{IR}}=1.3\left(S_{12}+S_{25}\right)+0.7\left(S_{25}+S_{60}\right)+0.2\left(S_{60}+S_{100}\right)$ and $S_{i}$ is the flux density in the IRAS band $i$ expressed in Jy. We obtained $L_{\mathrm{IR}} \sim(7.9 \pm 1.6) \times 10^{3} L_{\odot}$.

Adopting standard parameters for dust grains (Draine \& Lee 1984), the dust temperature can be derived from the relation $T_{\mathrm{d}}(\mathrm{K})=\left(95.94 / \mathrm{ln} B_{n}\right)$, where $B_{n}=1.667^{3+n} S_{100} / S_{60}$
Table 1. 2MASS sources towards IRAS 18544+0112.

\begin{tabular}{ccccccc}
\hline \hline No. & $l$ & $b$ & 2MASS & $J$ & $H$ & $K_{\mathrm{S}}$ \\
\hline 1 & 34.69 & -0.64 & $18565828+0116334$ & 15.73 & 15.18 & 14.37 \\
2 & 34.70 & -0.65 & $18570030+0116478$ & 18.59 & 15.65 & 13.35 \\
3 & 34.69 & -0.65 & $18565878+0116233$ & 17.76 & 14.59 & 12.37 \\
4 & 34.69 & -0.65 & $18565848+0116162$ & 17.04 & 15.89 & 15.13 \\
5 & 34.69 & -0.65 & $18565987+0116116$ & 16.71 & 15.71 & 15.66 \\
6 & 34.68 & -0.65 & $18565808+0115548$ & 15.27 & 11.58 & 9.76 \\
7 & 34.69 & -0.65 & $18565816+0116025$ & 18.04 & 15.69 & 13.97 \\
8 & 34.69 & -0.64 & $18565802+0116298$ & 14.44 & 13.86 & 13.70 \\
9 & 34.69 & -0.64 & $18565665+0116434$ & 17.46 & 13.94 & 12.10 \\
10 & 34.70 & -0.65 & $18565913+0116360$ & 14.25 & 13.78 & 13.55 \\
11 & 34.70 & -0.64 & $18565855+0116541$ & 17.14 & 15.31 & 14.50 \\
12 & 34.69 & -0.64 & $18565794+0116215$ & 16.10 & 15.19 & 14.21 \\
13 & 34.69 & -0.65 & $18565971+0115541$ & 15.11 & 11.38 & 9.49 \\
\hline
\end{tabular}

is the modified Planck function, and $\mathrm{n}$ is a parameter related to the absorption efficiency of the dust $\left(\kappa_{v} \alpha v^{n}\right)$. We obtained $T_{\mathrm{d}} \sim(29.0 \pm 5.0) \mathrm{K}$ for the adopted value $n=1$. The estimated dust temperature of about $29 \mathrm{~K}$ for IRAS $18544+0112$ is quite low for a UCHII region, which typically has temperatures of about $200 \mathrm{~K}$ (Ball et al. 1996), while it agrees with the ones derived for high mass protostellar candidates by Sridharan et al. (2002).

In summary, from the observed characteristics we suggest that IRAS $18544+0112$ is not yet a UCHII region.

\section{A search of MYSO candidates in IRAS 18544+0112}

To search for MYSO candidates associated with IRAS 18544+0112 we performed a near-infrared photometric analysis of all the sources that are enclosed within the borders of the IR source as seen in the $8 \mu \mathrm{m}$ band (see e.g. Fig. 2). We used the 2MASS All-Sky Point Source Catalogue (Skrutskie et al. 2006) in bands $J(1.25 \mu \mathrm{m}), H(1.65 \mu \mathrm{m})$ and $K_{\mathrm{S}}(2.17 \mu \mathrm{m})$, selecting only the sources detected in at least two bands. We found 13 sources in this region which are listed in Table 1 and shown in Figs. 6a-c. In Table 1 we present the source numbers (same as in the following figures), the Galactic coordinates in degrees, the Two Micron All Sky Survey (2MASS) designation and the $J, H$ and $K_{\mathrm{S}}$ magnitudes in Cols. 1 to 7 , respectively. Figures $6 \mathrm{a}-\mathrm{c}$ display the spatial location of these sources superimposed over: the $8 \mu \mathrm{m}$ emission (a), the near infrared $J H K$ three-color image extracted from the 2MASS (b), and the optical emission as extracted from the 2nd Digitized Sky Survey Blue (DSS2-B) (c). The dashed circle represents the area in which we searched for the mentioned sources.

Figures 7 and 8 display the $(H-K \mathrm{~s})$ versus $(J-H)$ color-color (CC) diagram and the $(H-K \mathrm{~s})$ versus $K$ s color-magnitude $(\mathrm{CM})$ diagram, respectively, of the 13 selected 2MASS sources.

Figure 7 shows that sources one to four, seven and twelve lie in the region of sources with infrared excess. In particular sources two and three appear as the most reddened ones. Sources six, nine, eleven and thirteen correspond to normally reddened main sequence stars and sources eight and ten, the most conspicuos ones in the optical image (Fig. 6c), are probably no reddened foreground stars.

According to the CM diagram (Fig. 8), sources two, three, and nine are the earliest spectral type stars in the region. Sources six and, thirteen lie in the region of the giant stars, and sources one, four, five, seven, eight, ten, eleven, and twelve correspond 

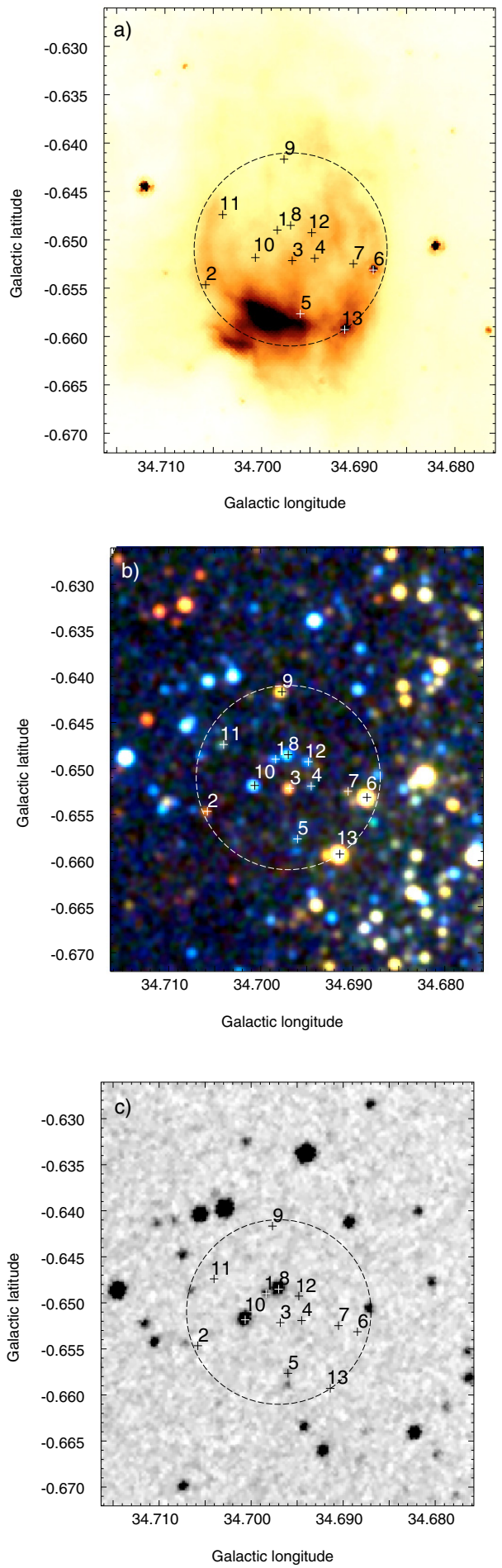

Fig. 6. Spatial location of the 2MASS sources found towards IRAS $18544+0112$ over the $8 \mu \mathrm{m}$ emission a), the near infrared $J H K$ three-color image extracted from The Two Micron All Sky Survey b) and the DSS2-B optical image c). The dashed circle represents the area in which we searched for the mentioned sources.

to spectral-type stars later than B3. Moreover, as Fig. 6c shows, sources one, five, eight, and ten are detected in the DSS2-B optical band which, given the high obscuration of the region, suggests that they are probably foreground stars.

Thus, based on both diagrams, we conclude that the most likely high mass protostars related to IRAS 18544+0112 would be sources two and three. The fact that source three lies towards

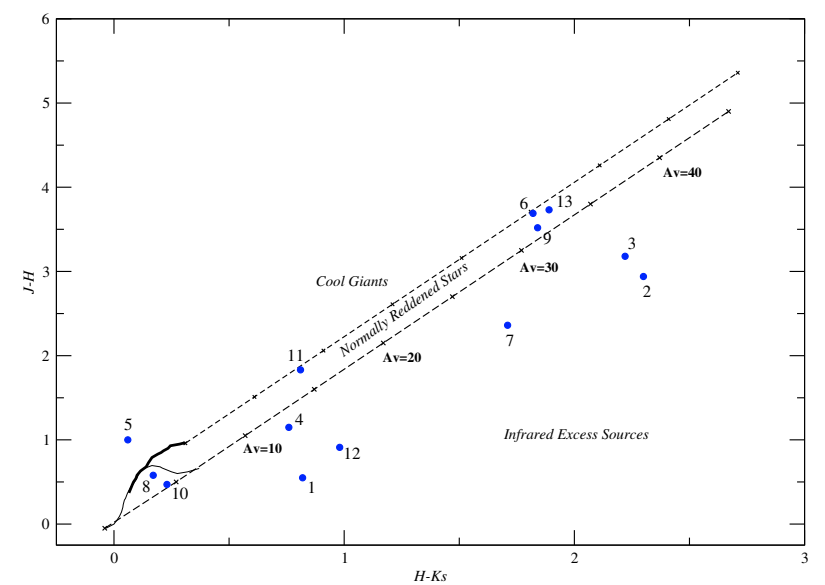

Fig. 7. Color-color diagram of the 13 2MASS sources found towards IRAS 18544+0112. The two solid curves represent the location of the main sequence (thin line) and the giant stars (thicker line) derived from Bessell \& Brett (1988). The parallel dashed lines are reddening vectors with the crosses placed at intervals corresponding to five magnitudes of visual extinction. We have assumed the interstellar reddening law of Rieke \& Lebofsky (1985) $\left(A_{J} / A_{V}=0.282 ; A_{H} / A_{V}=0.175\right.$ and $\left.A_{K} / A_{V}=0.112\right)$. The plot is classified into three regions: cool giants, normally reddened stars and infrared excess sources. The numbers correspond to the numbered sources of Figs. $6 \mathrm{a}-\mathrm{c}$.

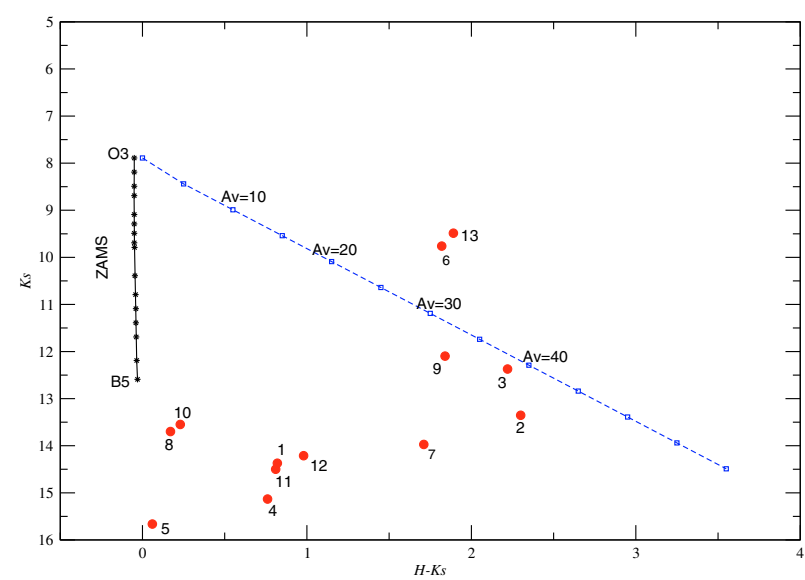

Fig. 8. Color-magnitude diagram of the 13 2MASS infrared sources towards IRAS 18544+0112. The solid curve represents the position of the main sequence at a distance of $3 \mathrm{kpc}$. The reddening vector for an $\mathrm{O} 3$ star, with the squares placed at intervals corresponding to five magnitudes of visual extinction, is shown with a dashed line. The numbers correspond to the numbered sources of Figs. $6 \mathrm{a}-\mathrm{c}$.

the geometrical center of the IR nebula and that it is placed on a maximun of the $24 \mu \mathrm{m}$ emission while source two is located onto the nebulas's border, suggests that source three is the main one responsible for the observed infrared nebula.

\section{Possible scenario}

In this section we discuss a possible formation scenario for IRAS 18544+0112.

As mentioned in Sect. 2, the presence of a PDR bordering the HII region G034.8-0.7 together with the observed morphology of its associated molecular cloud, are clear evidence of the fact that the HII region perturbs its enviroment.

The IR source IRAS $18544+0112$ is seen in projection inside the HII region G034.8-0.7, while IRAS 18542+0114 


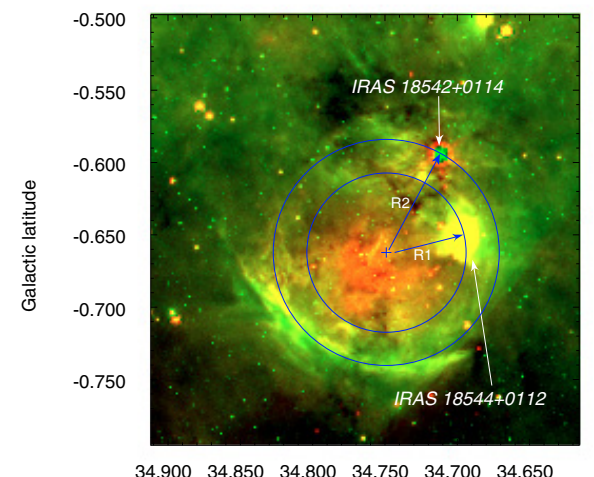

Galactic longitude

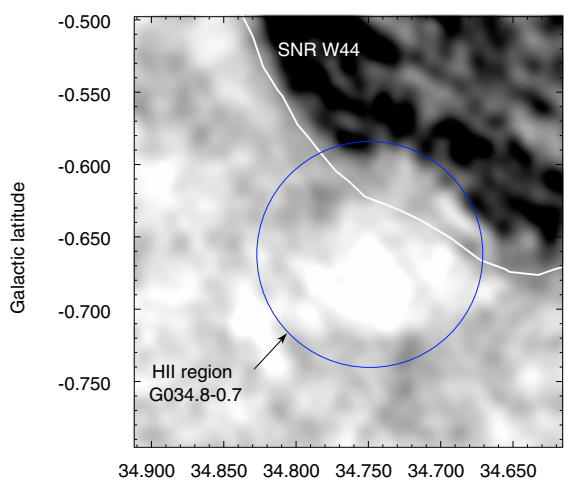

Galactic longitude
Fig. 9. Left: Two-color Spitzer image of the HII region G034.8-0.7 $(8 \mu \mathrm{m}=$ green and $24 \mu \mathrm{m}=$ red). The exterior and interior circles correspond to the size of the HII region at the moment it reached the location of IRAS 18542+0114 and IRAS 18544+0112, respectively. Right: tomographic map obtained between 74 and $324 \mathrm{MHz}$ (50" resolution). Black regions correspond to negative radio spectral indices, while greys and white indicate regions with a positive spectrum. Overlapped there is $1.4 \mathrm{HGz}$ radio continuum emission contour traced at $40 \mathrm{~K}$. The circle is the exterior one from the left pannel.
(Paron et al. 2009) is located upon its border (see Fig. 9). Based on an infrared and molecular study, Paron et al. (2009) found that IRAS $18542+0114$ is a MYSO with molecular outflow activity and whose formation was probably triggered by the expansion of G034.8-0.7 onto the molecular cloud. In this context, we suggest that the HII region G034.8-0.7 has also triggered the formation of IRAS $18544+0112$.

The observational differences found between both IRAS sources as well as their relative location with respect to the center of the HII region G034.8-0.7, suggest that the associated shockfront reached first the molecular gas where IRAS 18544+0112 was formed, thus explaining their different evolutionary stages. Kobayashi et al. (2008) proposed a similar scenario for the star forming region in Digel's Cloud 2, where an expanding shell has perturbed different parts of a molecular cloud during its evolution, producing different star generations.

To test the proposed scenario we estimate the age of the HII region at the moment it reached the location of each IRAS source. Using a simple model described by Dyson \& Williams (1980) we calculated the age of the HII region at a given radius $R$ as

$t(R)=\frac{4 R_{\mathrm{s}}}{7 c_{\mathrm{S}}}\left[\left(\frac{R}{R_{\mathrm{s}}}\right)^{7 / 4}-1\right]$

where $c_{\mathrm{S}}$ is the sound velocity in the ionized gas $\left(c_{\mathrm{S}}=10 \mathrm{~km} \mathrm{~s}^{-1}\right)$ and $R_{\mathrm{S}}$ is the radius of the Strömgren sphere, given by $R_{\mathrm{S}}=$ $\left(3 N_{\text {uv }} / 4 \pi n_{0}^{2} \alpha_{B}\right)^{1 / 3}$, where $\alpha_{B}=2.6 \times 10^{-13} \mathrm{~cm}^{3} \mathrm{~s}^{-1}$ is the hydrogen recombination coefficient to all levels above the ground level. $N_{\mathrm{uv}}$ is the total number of ionizing photons per unit of time emitted by the stars, and $n_{0}$ is the original ambient density.

The number $N_{\mathrm{uv}}$ of UV ionizing photons needed to keep an HII region ionized is given by (see, e.g. Chaisson 1976) $N_{\mathrm{uv}}=0.76 \times 10^{47} T_{4}^{-0.45} v_{\mathrm{GHz}}^{0.1} S_{v} D_{\mathrm{kpc}}^{2}$, where $T_{4}$ is the electron temperature in units of $10^{4} \mathrm{~K}, D_{\mathrm{kpc}}$ the distance in kpc, $v_{\mathrm{GHz}}$ the frequency in $\mathrm{GHz}$, and $S_{v}$ the measured total flux density in Jy. To calculate the radio flux density and in order to overcome the problem of superposition of the thermal radiation of the HII region and the non-thermal flux of the SNR that occurs in the western half of the HII region, we assumed the HII region to consist of two identical halves; we therefore integrated the flux density over the "free" eastern half of the ionized sphere and assumed that for the complete region it is just twice this value. Using the $1.4 \mathrm{GHz}$ VLA image we estimated $S_{1.4 \mathrm{GHz}} \sim 3 \mathrm{Jy}$, which agrees with previous estimations, $S_{4.85 \mathrm{GHz}}=2.6 \mathrm{Jy}$ (Kuchar \& Clark 1997), and $S_{2.7 \mathrm{GHz}}=2.8 \mathrm{Jy}$ (Paladini et al. 2003) for a spectral index $\alpha=-0.1\left(S_{v} \sim v^{\alpha}\right)$ typical for the optically thin regime of HII regions. For an HII region with $T=10^{4} \mathrm{~K}$ placed at a distance of $3 \mathrm{kpc}$, the total amount of ionizing photons needed to keep the source ionized turns out to be about $N_{\mathrm{uv}}=1.7 \times 10^{48} \mathrm{ph} \mathrm{s}^{-1}$. Based on the ionizing fluxes for massive stars given by Schaerer \& de Koter (1997), we infer that the ionizing star cannot be later than $09.5 \mathrm{~V}$. However, this is only a coarse limit. The exciting star is probably earlier than 09.5 since if the observed infrared emission originates in dust heated by stellar radiation, part of the UV radiation is dissipated in this way.

To estimate the dynamical age of the HII region it is important to determine the center from which the ionized gas is expanding. Based on the infrared and radio continuum emission distribution we identify an almost circular morphology for the HII region G034.8-0.7. Figure 9 (left) shows that infrared emission at $8 \mu \mathrm{m}$ (green) clearly delineates the southeastern border of the HII region, while the border where IRAS $18542+0114$ is located is diffuse and fainter. To better delineate this border we inspected a tomographic map traced between 74 and $324 \mathrm{MHz}$ towards W44 (see Fig. 9 (right)). In a radio study of SNR W44, Castelletti et al. (2007) found a curious spectral index inversion on the southeastern limb of W44 that appears as an indentation in the SNR boundary. There, the spectrum changes from a negative value corresponding to the SNR synchrotron radiation (spectral index $\alpha \sim-0.6$ ) to $\alpha \geq 0$. In Fig. 9 (right) darker regions correspond to a negative spectral index. We propose that the spectral inversion must be the result of free-free absorption produced by thermal ionized gas naturally explained by the presence of the HII region G034.8-0.7 located between us and the SNR. In this way, we can delineate the northwestern border of the HII region where IRAS $18542+0114$ is located, thus determining the probable center of the expanding HII region.

By adopting the radii $R_{1}=3 \mathrm{pc}$ and $R_{2}=4 \mathrm{pc}$ for IRAS 18544+0112 and IRAS 18542+0114, respectively, an ambient density in the region of less than $\sim 10^{3} \mathrm{~cm}^{-3}$ and as the exciting agent for the HII region an 09.5 star, we derived dynamical ages of about $0.6 \times 10^{6}$ and $1.2 \times 10^{6}$ yr for the HII region at the position of IRAS $18544+0112$ and IRAS $18542+0114$, respectively. An age difference of $\sim 6 \times 10^{5} \mathrm{yr}$ is compatible with the observed different evolutive stages of both IR sources.

The remaining question is whether the expansion of the SNR W44 had some influence in the processes observed in this molecular complex. Based on the age of the associated pulsar, Wolszczan et al. (1991) estimated an age of about $2 \times 10^{4} \mathrm{yr}$ 
for the SNR W44. Thus, the possibility that the SNR W44 has triggered the star formation in this region seems unlikely.

\section{Summary}

We present a molecular and infrared analysis of the IR source IRAS $18544+0112$. The main results can be summarized as follows:

(a) We find ${ }^{12} \mathrm{CO} J=3-2$ self-absorbed profiles, which are typical of star forming regions. However, we do not detect any evidence of an outflow activity in IRAS 18544+0112 either in the molecular lines or in the infrared emission distribution.

(b) The analysis of the ${ }^{12} \mathrm{CO} J=3-2$ line suggests the presence of expanding molecular gas in the region.

(c) Based on its morphology, infrared and molecular parameters, and the non detection of ionized gas, we suggest that IRAS $18544+0112$ is an evolved high mass protostellar object which has not yet reached the ultracompact HII region stage.

(d) From a near-infrared photometric analysis of the point sources observed towards IRAS $18544+0112$ we propose that 2MASS $18565878+0116233$ source (source three in the text) is the MYSO candidate most likely associated with IRAS $18544+0112$.

(e) Based on the observational evidence that the HII region G034.8-0.7 is perturbing the neighboring molecular clouds we suggest that it has triggered at least two star forming regions, IRAS 18542+0114 and IRAS 18544+0112, during its expansion.

Acknowledgements. S.P. is grateful to the staff of ASTE for the support received during the observations, especially to Juan Cortés. S.P. acknowledges the support of Viviana Guzmán during the observations. M.O. is a doctoral fellow of CONICET, Argentina. S.P., S.C., G.C., and G.D. are members of the Carrera del Investigador Científico of CONICET, Argentina. This work was partially supported by Argentina grants awarded by CONICET, UBA and ANPCYT. M.R. is supported by the Chilean Center for Astrophysics FONDAP No. 15010003. M.R. and S.P. acknowledge support from FONDECYT $\mathrm{N}^{\circ}$ 1080335. This work has made use of GLIMPSE and MIPSGAL data obtained with the Spitzer Space Telescope, which is operated by the Jet Propulsion Laboratory, California Institute of Technology, under NASA contract 1407. We also used data products from the Two Micron All Sky Survey, the NASA/IPAC Infrared Science Archive, which is operated by the Jet Propulsion Laboratory, California Institute of Technology, under contract with NASA. We are grateful to the anonymous referee, whose comments and suggestions led to the improvement of this Paper.

\section{References}

Ball, R., Meixner, M. M., Keto, E., Arens, J. F., \& Jernigan, J. G. 1996, AJ, 112, 1645

Bessell, M. S., \& Brett, J. M. 1988, PASP, 100, 1134

Castelletti, G., Dubner, G., Brogan, C., \& Kassim, N. E. 2007, A\&A, 471, 537

Cesarsky, D., Lequeux, J., Abergel, A., et al. 1996, A\&A, 315, L309

Chaisson, E. J. 1976, in Frontiers of Astrophysics, 259

Chan, G., \& Fich, M. 1995, AJ, 109, 2611

Comerón, F., Schneider, N., \& Russeil, D. 2005, A\&A, 433, 955

Condon, J. J., Cotton, W. D., Greisen, E. W., et al. 1998, AJ, 115, 1693

Cyganowski, C. J., Whitney, B. A., Holden, E., et al. 2008, AJ, 136, 2391

Deharveng, L., Zavagno, A., \& Caplan, J. 2005, A\&A, 433, 565

Draine, B. T., \& Lee, H. M. 1984, ApJ, 285, 89

Dyson, J. E., \& Williams, D. A. 1980, Physics of the interstellar medium (New York: Halsted Press), 204

Elmegreen, B. G., \& Lada, C. J. 1977, ApJ, 214, 725

Ezawa, H., Kawabe, R., Kohno, K., \& Yamamoto, S. 2004, in SPIE Conf. Ser. 5489, ed. J. M. Oschmann, Jr., 763

Guan, X., Wu, Y., \& Ju, B. 2008, MNRAS, 391, 869

Hogerheijde, M. R., van Dishoeck, E. F., Blake, G. A., \& van Langevelde, H. J. 1998, ApJ, 502, 315

Hughes, V. A., \& MacLeod, G. C. 1994, ApJ, 427, 857

Jackson, J. M., Rathborne, J. M., Shah, R. Y., et al. 2006, ApJS, 163, 145

Johnstone, D., Boonman, A. M. S., \& van Dishoeck, E. F. 2003, A\&A, 412, 157

Kobayashi, N., Yasui, C., Tokunaga, A. T., \& Saito, M. 2008, ApJ, 683, 178

Kuchar, T. A., \& Clark, F. O. 1997, ApJ, 488, 224

Kumar, M. S. N., \& Grave, J. M. C. 2007, A\&A, 472, 155

Leger, A., \& Puget, J. L. 1984, A\&A, 137, L5

Lehtinen, K. 1997, A\&A, 317, L5

Leung, C. M. 1978, ApJ, 225, 427

Melioli, C., de Gouveia Dal Pino, E. M., de La Reza, R., \& Raga, A. 2006, MNRAS, 373, 811

Molinari, S., Brand, J., Cesaroni, R., \& Palla, F. 1996, A\&A, 308, 573

Paladini, R., Burigana, C., Davies, R. D., et al. 2003, A\&A, 397, 213

Paron, S., Ortega, M. E., Rubio, M., \& Dubner, G. 2009, A\&A, 498, 445

Pomarès, M., Zavagno, A., Deharveng, L., et al. 2009, A\&A, 494, 987

Rawlings, J. M. C., Redman, M. P., Keto, E., \& Williams, D. A. 2004, MNRAS, 351,1054

Rieke, G. H., \& Lebofsky, M. J. 1985, ApJ, 288, 618

Schaerer, D., \& de Koter, A. 1997, A\&A, 322, 598

Skrutskie, M. F., Cutri, R. M., Stiening, R., et al. 2006, AJ, 131, 1163

Sridharan, T. K., Beuther, H., Schilke, P., Menten, K. M., \& Wyrowski, F. 2002, ApJ, 566, 931

Stil, J. M., Taylor, A. R., Dickey, J. M., et al. 2006, AJ, 132, 1158

Vanhala, H. A. T., \& Cameron, A. G. W. 1998, ApJ, 508, 291

Whitworth, A. P., Bhattal, A. S., Chapman, S. J., Disney, M. J., \& Turner, J. A. 1994a, A\&A, 290, 421

Whitworth, A. P., Bhattal, A. S., Chapman, S. J., Disney, M. J., \& Turner, J. A. 1994b, MNRAS, 268, 291

Wolszczan, A., Cordes, J. M., \& Dewey, R. J. 1991, ApJ, 372, L99

Zavagno, A., Deharveng, L., Comerón, F., et al. 2006, A\&A, 446, 171

Zhou, S. 1992, ApJ, 394, 204

Zhou, S., Evans, II, N. J., Koempe, C., \& Walmsley, C. M. 1993, ApJ, 404, 232 